Accepted refereed manuscript of: Fedurek P, Slocombe KE \& Zuberbühler K (2015) Chimpanzees communicate to two different audiences during aggressive interactions. Animal Behaviour, 110, pp. 21-28. DOI: https://doi.org/10.1016/j.anbehav.2015.09.010

(c) 2015, Elsevier. Licensed under the Creative Commons Attribution-NonCommercial-NoDerivatives 4.0 International

http://creativecommons.org/licenses/by-nc-nd/4.0/

\title{
1 Chimpanzees communicate to two different audiences during aggressive interactions
}

2 Pawel Fedurek ${ }^{a}{ }^{*}$, Katie E. Slocombe ${ }^{\text {b }}$, Klaus Zuberbühler ${ }^{\text {a’c }}$

b Department of Psychology, University of York, York, U.K ${ }^{\mathrm{c}}$ School of Psychology and

5 Neuroscience, University of St Andrews, St Andrews, U.K

$8 \quad$ Received 3 June 2015

$9 \quad$ Initial acceptance 4 August 2015

10 Final acceptance 21 August 2015

11 MS. number: 15-00475

12 *Correspondence: P. Fedurek, Institute of Biology, University of Neuchâtel, Rue Emile-

13 Argand 11, 2000 Neuchâtel, Switzerland.

14 E-mail address: fedurek@ hotmail.co.uk (P. Fedurek).

16 Conflict and aggressive interactions are common phenomena in group-living animals and vocal behaviour often plays an important role in determining their outcomes. In some species, vocal signals seem to provide bystanders with information about the nature of an ongoing aggressive interaction, which can be beneficial for the victims. For example, in chimpanzees and some other primates, victims adjust their screams depending on the composition of the by-standing audience, probably to solicit their support. Considerably less is known, however, 
22 about the role of other call types produced by victims of aggression. In this study, we focused on the fact that, immediately after screams, chimpanzee, Pan troglodytes schweinfurthii, victims often produce 'waa' barks, but little is known about their function. Our results showed that for screams, but not 'waa' barks, production was dependent on the audience composition with victims being more likely to scream when adult or late-adolescent males were in close proximity. We also found that after 'waa' barking, but not screaming, victims were more likely to retaliate against and less likely to reconcile with their aggressors, and that 'waa' barking was more common after victims had received support from other party members. These results suggest that, in chimpanzees, victims of aggression vocalize with a dual social strategy of attempting to recruit support from bystanders and to repel their attackers by signalling readiness to retaliate. We conclude that victim scream and 'waa' bark calls, although often produced during the same agonistic event, are directed at different audiences and fulfil different social functions, and that these calls can mediate both aggressive interactions and aggressor-victim relationships following aggression. 
Agonistic interactions are a common consequence of group living (Nieburg, 1970), which can bring about substantial costs to the opponents, including severe injuries, mutilations or death. One way to minimize the costs of aggressive interactions is for opponents to communicate their behavioural intentions in order to prevent costly escalations (Smith, 1977). For example, an opponent can signal submission or willingness to retaliate or recruit support from bystanders, with vocal behaviour playing a key role in achieving these goals.

During animal conflicts screams are probably the most common vocalizations and various functions have been attributed to them, such as alerting group members, confusing or dissuading the opponent or attracting help (Hogstedt, 1983; Rohwer, Fretwell, \& Tuckfield, 1976). In primates, screams are commonly produced by victims of aggression, apparently to alert and recruit aid from allies (Bernstein \& Ehardt, 1985; Cheney, 1977; Gouzoules, Gouzoules, \& Marler, 1984). For example, rhesus macaques, Macaca mulatta, produce acoustically distinct variants of screams that seem to be related to the identity of the caller, the dominance rank of the opponent, the relatedness between the caller and opponent and the severity of the attack (Gouzoules \& Gouzoules, 1990; Gouzoules et al., 1984). Receivers attend differently to different scream variants, suggesting that the calls inform potential supporters about the nature of the aggressive interaction (Gouzoules et al., 1984).

In chimpanzees, Pan troglodytes schweinfurthii, recruiting support from bystanders also seems to be an important function of screams. Here, the acoustic structure varies as a function of the severity of the aggression (Slocombe \& Zuberbühler, 2007) and these differences seem to be informative for the receiver (Slocombe, Townsend, \& Zuberbühler, 2009). Victims and aggressors produce acoustically different screams (Slocombe \& Zuberbühler, 2005) enabling the receiver to infer something regarding the nature of the aggressive encounter (Slocombe, Kaller, Call, \& Zuberbühler, 2010). Importantly, screams are individually distinctive (Kojima, Izumi, \& Ceugniet, 2003) and victims of aggression can modify the acoustic 
structure of their screams to exaggerate the aggression received if individuals of equal or higher rank to the opponent are nearby, which is likely to increase the probability of receiving aid (Slocombe \& Zuberbühler, 2007).

In chimpanzees, however, victims of aggression often produce another type of call, 'waa' barks. Chimpanzee 'waa' barks belong to an acoustic cluster of bark vocalizations that are given in several contexts, such as hunting or when replying to long-distance calls from other group members or from members of other communities (Crockford \& Boesch, 2003;

Goodall, 1986; Marler \& Tenaza, 1977). 'Waa' barks are also given to alert others about predators (Crockford \& Boesch, 2003; Schel, Townsend, Machanda, Zuberbühler, \& Slocombe, 2013) or to drive away dangerous animals, such as bush pigs (P. Fedurek, personal observation), suggesting that, although these calls can have subtly different acoustic structure depending on the context of production (Crockford \& Boesch, 2003), they are linked to targeted aggressive motivation. 'Waa' barks are also given in agonistic encounters and it has been proposed that they are signals directed at aggressors (Goodall, 1986; Marler \& Tenaza, 1977), usually given immediately after screams from which they can grade (Marler, 1976; Marler \& Tenaza, 1977). Overall, however, there has been little systematic analysis of the function of this call type in agonistic contexts. One notable exception concerns the observation that, during agonistic interactions, 'waa' barks are sometimes given by allies of the opponents observing the interaction, possibly as a way of expressing support (NewtonFisher, 2006; Wittig, Crockford, Langergraber, \& Zuberbühler, 2014).

The aim of this study was to examine the function of victim 'waa' barks and to investigate how victim screams and barks are deployed during aggressor-victim interactions. We hypothesized that 'waa' barks are optional signals directed at the aggressor in specific situations to signal the probability of retaliation. 
87 To address our hypothesis, we tested the following predictions. First, if 'waa' barks were directed at the aggressor rather than a third-party audience, we expected that, in contrast to screams, 'waa' bark production would be independent of the audience composition. We therefore compared the production of both call types as a function of the number of males or females in the party and the presence of at least one affiliated or higher-ranking group member in close proximity to the victim $(<15 \mathrm{~m})$ or within the party (e.g. Fedurek \& Slocombe, 2013). Second, we predicted that if 'waa' barks were directed at aggressors, victims should be visually oriented towards their aggressors during call production. If 'waa' barking signalled the probability of retaliation, we predicted that utterances containing 'waa' barks would be associated with higher rates of retaliation and lower rates of reconciliation with the aggressor compared to utterances with screams only. Finally, if 'waa' barks expressed aggressive motivation, we predicted that victims would be more likely to produce these signals after rather than before receiving support from third-party individuals, when the risk of renewed aggression from the aggressor is low.

$<\mathrm{H} 1>$ Methods

\section{$\leq \mathrm{H} 2>$ Study site and study subjects}

The study was conducted with the Sonso chimpanzee community of Budongo Forest, Uganda. The group has been under constant observation since 1990 and is well habituated to the presence of human observers (Reynolds, 2005). At the time of the study, the community contained 75 individuals with a home range of around $15 \mathrm{~km}^{2}$. Study subjects were adult males and females ( $N=11: \geq 16$ years; $N=24: \geq 15$ years; (Goodall, 1986)) and adolescents 
110 ( $N=3$ early males: $8-12$ years; $N=3$ late males: $13-15$ years; $N=9$ early females: $8-10$ years 111 old; $N=4$ late females: $11-14$ years).

$\leq \mathrm{H} 2>$ Sampling method

This study was approved by the Institute of Biology Ethics Committee at the University of

Neuchâtel and permission to conduct the study was granted by the Uganda Wildlife Authority and the Uganda National Council for Science and Technology. The study was conducted between June and October 2013, February and September 2014 and January and April 2015. Data were collected between 0700 and 1630 hours local time. Since agonistic interactions were relatively rare, we used all-occurrence sampling (Altmann, 1974). For each aggressive interaction we recorded (1) the identity of the aggressor and victim, (2) the type of aggression, (3) whether or not the victim called and the type of calls given, (4) whether the victim was oriented towards the aggressor if 'waa' barking occurred, (5) the closest distance between aggressor and victim at the beginning of screaming and 'waa' barking, (6) the identities of all audience members within $15 \mathrm{~m}$ (relative to the victim at the beginning of aggression), (7) whether or not the victim or aggressor received support from bystanders, (8) whether or not there was a reconciliation between the aggressor and the victim, and (9) whether or not the victim retaliated against the aggressor (see section below for definitions of these behaviours).

In addition, a randomly chosen focal adult or late-adolescent male was followed continuously every day of data collection to obtain data on party composition and male preferred social partners. Instantaneous scan samples (Altmann, 1974) at 15 min intervals were conducted to record (1) the identities of individuals present in the focal individual's party (defined as all 
adult and late-adolescent individuals present within $35 \mathrm{~m}$ of the focal animal; Newton-Fisher, 1999), (2) the identities of individuals present within $5 \mathrm{~m}$ of the focal male and (3) the identity of the adult or late-adolescent individual closest to the focal male.

\section{$\underline{\mathrm{H} 2>\text { Data recorded and definitions }}$}

\section{$<\mathrm{H} 3>$ Screams and 'waa' barks}

For every act of aggression in the focal party, we noted whether or not the victim produced screams and whether or not these were followed by 'waa' barks (within 10 min of the end of aggression). Although these two types of calls often grade from one to another, they are acoustically distinguishable. 'Waa' barks have an abrupt onset, are typically shorter, and have a lower frequency range and a noisier spectral quality than screams (Fig. 1; Crockford \& Boesch, 2003). The call typically starts with a low-frequency ' $w$ ' introductory phase and culminates with a higher frequency element usually sounding to the human ear as an 'aow' or 'aoo' sound (Schel et al., 2013). In agonistic contexts, 'waa' barks usually grade from screams and occur either immediately after the last call of a scream bout or within a scream bout, in which case they are both preceded and followed by screams (Fig. 1; see Supplementary material Audio S1 and Audio S2 for examples of recordings). We recorded the presence or absence of screams and waa barks during and after each agonistic interaction in real time. High-quality audio recordings were available for a small number of the agonistic events observed and all calls $(N=142)$ from these 16 events were categorized from these audio recordings independently by P.F., K.S. and an independent coder, who was blind to the hypotheses and aims of the study but trained in categorizing chimpanzee calls. There was $100 \%$ agreement between the three coders on the classification of these calls as screams ( $N=$ 
159 124) or 'waa' barks $(N=18)$, indicating that these calls were reliably distinguished in the 160 field.

161

$<\mathrm{H} 3>$ Severe and mild aggression

163 We distinguished between two categories of aggression depending on its severity. Severe aggression took place when the aggressor physically attacked the victim (slap, kick, bite, etc.) or when the victim was chased by the aggressor (i.e. the pursuit distance was more than $7 \mathrm{~m}$ ) but there was no physical contact between them. Mild aggression was defined as instances of aggression such as charge (i.e. the pursuit distance was less than $7 \mathrm{~m}$ ), displaying towards another individual (i.e. a male runs piloerect towards another individual, and may include shaking vegetation, slapping the ground (Goodall, 1986)), and postural threat such as arm raises or ground slaps directed at the victim (Slocombe \& Zuberbuhler, 2007).

$<\mathrm{H} 3>$ Audience

We determined all adult and late-adolescent males or females in close proximity to the victim (<15 $\mathrm{m}$ away) at the start of aggression. Data on adult and late-adolescent individuals present in the victim's party were taken from the 15 min scan preceding the aggression.

$<\mathrm{H} 3>$ Retaliation

Retaliation was defined as the victim directing mild or severe aggression towards the aggressor within 10 min after the agonistic interaction had terminated.

$<\mathrm{H} 3>$ Support for victim

Support for the victim took place when one or more individuals aided the victim by directing mild or severe aggression towards the aggressor (e.g. Mitani \& Gros-Louis, 1998). 
$<\mathrm{H} 3>$ Reconciliation

Reconciliation between aggressors and victims took place when there was an affiliative interaction, such as sitting in contact, allogrooming, presenting, mounting, genital inspection, embracing, gentle touching or soft biting (Arnold \& Whiten, 2001) between the two opponents within 10 min of the end of aggression (e.g. de Waal \& Yoshihara, 1983).

Reconciliation was also considered to have taken place if during that $10 \mathrm{~min}$ period there was a prolonged (i.e. for at least $10 \mathrm{~s}$ ) close proximity (i.e. equal to or less than $1 \mathrm{~m}$ ) between the former aggressor and victim initiated by ether of the opponents (e.g. Aureli, Cords, \& van Schaik, 2002; McFarland \& Majolo, 2013).

$<\mathrm{H} 3>$ Victim orientation during 'waa' barking

During 'waa' barking, the victim was oriented towards the aggressor when the victim's face was directed towards the aggressor rather than in other directions.

$<\mathrm{H} 3>$ Preferred social partners

Preferred social partners (PSPs) were identified only for adult and late-adolescent males. PSPs were established on the basis of three different dyadic association measures: simple ratio index (time spent in a party together), $5 \mathrm{~m}$ association index and nearest-neighbour association index (Gilby \& Wrangham, 2008; see Appendix).

Dominance status was established only for adult and late-adolescent males, using the Elorating procedure (Neumann et al., 2011; see Appendix). Rank difference between two male opponents was established by deducting the rank of the aggressor from the rank of the victim. 
211 Generalized linear mixed-effect models (GLMM) and linear mixed-effect models (LMM)

212 were used in all the analyses. In all analyses each aggression event was entered as one data

213 point. To avoid the problem of nonindependence of data (e.g. Waller, Warmelink, Liebal,

214 Micheletta, \& Slocombe, 2013), we incorporated in the analyses data on entities from which repeated measurements were taken as 'random effects', which in our models concerned the identities of the aggressor and the victim. All statistical analyses were conducted using STATA 12.0 software (StataCorp LP, College Station, TX, U.S.A.).

$<\mathrm{H} 3>$ Models created

In the majority of models the entire data set was used. However, for the analyses concerning dominance rank and PSPs, we used data only on adult and late-adolescent males, for whom we had accurate data on dominance and affiliation relationships.

To examine whether the production of screams and 'waa' barks was predicted by the severity of aggression and the sex of the victim, we created two GLMMs in which we put as the dependent variable whether or not (0/1) screams or 'waa' barks occurred during aggression, and as independent variables the type of aggression (0: mild; 1 : severe) and the sex of the

227 victim (0: female; 1: male). Only data from adult and late-adolescent males and females, 228 which were the most common age-sex categories of the victim and aggressor in our data set 229 (Table 1), were incorporated in this analysis $(N=216)$.

230 To examine whether the production of screams and 'waa' barks was predicted by the distance in terms of dominance rank between the victim and the aggressor, we created two GLMMs, 
in which we put as the dependent variable whether or not (0/1) screams or 'waa' barks occurred during aggression, and as the independent variable the rank distance between the victim and the aggressor. Only data from adult and late-adolescent males, for whom we had accurate dominance data, were considered in this analysis $(N=130)$.

To examine whether there was a difference in terms of the distance between the aggressor and the victim during screaming and waa barking, we created an LMM in which we put as the dependent variable the closest distance $(\mathrm{m})$ between the victim and aggressor during calling and as the independent variable whether the call was a scream (0) or a 'waa' bark (1). Since data points with 'waa' barks $(N=56)$ also contained screams, in this model aggression bout ID was set as another random effect in addition to aggressor ID and victim ID. For this analysis, we only included data from aggressive bouts in which either screams or 'waa' barks were produced $(N=195)$.

To examine the effect of audience both in close proximity to the victim and in the victim's party on the probability of screaming or 'waa' barking, we created two GLMMs in which we put as the dependent variable the occurrence (0/1) of screams or 'waa' barks, and as independent variables (1) the number of males in close proximity to the victim, (2) the number of females in close proximity to the victim, (3) the total number of males in the party and (4) the total number of females in the party $(N=223)$.

To investigate whether the presence of a PSP or a higher ranking individual predicted screaming or 'waa' barking, we created two models in which we put either scream $(0 / 1)$ or 'waa' bark (0/1) as the dependent variable, and the presence of a PSP (0: non-PSP; 1: PSP) and an individual that outranked the aggressor (0: lower ranking; 1: higher ranking) in both close proximity and the party. Only data on adult and late-adolescent males for whom accurate dominance and PSP data were available were considered in these analyses $(N=130)$. 
256 To investigate whether screams or 'waa' barks predicted the occurrence of the victim's

257 retaliation, we created a GLMM in which we put as the dependent variable whether or not

$258(0 / 1)$ retaliation occurred, and as independent variables the occurrence $(0 / 1)$ of screams and

259 'waa' barks. In this model we also put the type of aggression as another independent variable

260 to control for the effect of aggression type on the occurrence of retaliation $(N=223)$.

261 To examine whether the occurrence of screams or 'waa' barks predicted reconciliation

262 between the opponents, we created a GLMM in which we put as the dependent variable

263 whether or not $(0 / 1)$ there was reconciliation between the aggressor and the victim, and as

264 independent variables the presence of screams (0/1) and 'waa' barks (0/1). We also put the

265 type of aggression as another independent variable to control for the effect of aggression type

266 on the occurrence of reconciliation $(N=223)$.

267 To investigate whether screams or 'waa' barks were associated with audience support for the

268 victim, we created a GLMM in which we put as the dependent variable whether or not $(0 / 1)$

269 the victim received support from the audience, and as the independent variables the

270 occurrence of screams $(0 / 1)$ and 'waa' barks $(0 / 1)(N=223)$.

$<\mathrm{H} 1>$ Results

$<\mathrm{H} 2>$ Rates and context of victim screams and 'waa' barks

274 In total, we recorded 223 bouts of aggression (see Table 1 for the summary of data collected).

275 'Waa' barks were always produced during or after, but not before, screaming (56 of 56 'waa'

276 bark events). In $80 \%$ of cases $(N=45)$ 'waa' barks occurred during screaming or within $15 \mathrm{~s}$

277 after scream termination. For the remaining 20\% 'waa' barks occurred between $16 \mathrm{~s}$ and 10

278 min after the end of screaming. During 'waa' barking victims were always (all 56 events) 
visually oriented towards aggressors. In addition, while screams typically occurred during the exact time of assault (when the victim was charged, chased, physically attacked, etc.) lasting for up to several minutes after the first attack, 'waa' barks were never given during the act of aggression but usually $(91 \% ; N=51)$ shortly after the assault when the aggressor was still in visual contact. The mean closest distance between victims and aggressors was smaller during screaming (mean=3.45 m, SD=2.99) than during 'waa' barking (mean=10.52 m, SD=5.11; $\beta \pm \mathrm{SE}=7.13 \pm 0.53, z=13.52, P<0.001)$.

Victim screams occurred in $87 \%$ and 'waa' barks in $25 \%$ of all aggressive bouts $(N=223$;

Table 1). Screams $(\beta \pm \mathrm{SE}=3.31 \pm 1.37, z=2.41, P=0.016)$ and especially 'waa' barks $(\beta \pm \mathrm{SE}=1.18 \pm 0.40, z=2.94, P=0.003)$ were more likely to be produced in response to severe rather than mild aggression. When considering adult and late-adolescent individuals, males and females were equally likely to produce screams $(\beta \pm \mathrm{SE}=-1.10 \pm 0.68, z=-1.62, P=0.105)$ and 'waa' barks ( $\beta \pm \mathrm{SE}=0.58 \pm 0.41, z=1.41, P=0.155$; Table 1$)$. Rank difference between two male opponents did not predict the occurrence of 'waa' barks $(\beta \pm \mathrm{SE}=-0.08 \pm 0.09, z=-1.05$, $P=0.294)$. However, there was a nonsignificant trend showing that the larger the rank distance between the aggressor and the victim was, the more likely the victim was to produce screams $(\beta \pm \mathrm{SE}=0.23 \pm 0.13, z=1.76, P=0.078)$.

$<\mathrm{H} 2>$ Third-party audience effects on screaming and 'waa' barking

The production of screams was dependent on the number of adult and late-adolescent males, but not the number of adult and late-adolescent females, in close proximity $(<15 \mathrm{~m}$; Table 2 , Fig. 2). The number of males or females in the party $(<35 \mathrm{~m})$ had no effect (Table 2$)$. In contrast, 'waa' bark production was not affected by the number of males or females in close proximity or in the party (Table 3). 
Victims tended to scream $(\beta \pm \mathrm{SE}=2.48 \pm 1.50, z=1.65, P=0.099)$ but not 'waa' bark $(\beta \pm \mathrm{SE}=-$ $0.35 \pm 0.68, z=0.51, P=0.613)$ when an individual that was higher ranking than the aggressor was in close proximity. Victims were not more likely to scream $(\beta \pm \mathrm{SE}=-1.69 \pm 1.64, z=-1.03$, $P=0.302)$ or 'waa' bark $(\beta \pm \mathrm{SE}=0.08 \pm 0.74, z=0.10, P=0.917)$ when an individual that was higher ranking than the aggressor was in the victim's party.

Victims were not more likely to scream $(\beta \pm \mathrm{SE}=-2.49 \pm 1.68, z=-1.48, P=0.139)$ or 'waa' bark $(\beta \pm \mathrm{SE}=0.67 \pm 0.83, z=0.81, P=0.416)$ when a PSP of the victim was in close proximity.

Likewise, victims were not more likely to scream $(\beta \pm \mathrm{SE}=2.21 \pm 1.68, z=1.31, P=0.190)$ or 'waa' bark $(\beta \pm \mathrm{SE}=-1.02 \pm 0.74, z=-1.38, P=0.167)$ when a PSP of the victim was in the same party.

$<\mathrm{H} 2>$ Victims' calls and retaliation

'Waa' barking (Fig. 3; $\beta \pm \mathrm{SE}=3.18 \pm 1.44, z=2.20, P=0.028$ ) but not screaming $(\beta \pm \mathrm{SE}=14.72 \pm 1695.73, z=0.01, P=0.993)$ was associated with victims retaliating against the aggressor. The type of aggression did not predict the occurrence of retaliation $(\beta \pm \mathrm{SE}=1.77 \pm 1.35, z=1.31, P=0.190)$.

$<\mathrm{H} 2>$ Victims' calls and reconciliation

Reconciliation between aggressors and victims was less likely after 'waa' barking (Fig. 4; $\beta \pm \mathrm{SE}=-2.15 \pm 0.70, z=-3.09, P=0.002)$ but not screaming $(\beta \pm \mathrm{SE}=0.67 \pm 0.63, z=1.07, P=0.284)$.

The type of aggression did not predict the occurrence of reconciliation $(\beta \pm \mathrm{SE}=-0.49 \pm 0.49$, $z=-1.00, P=0.317)$. 
$<\mathrm{H} 2>$ Victims' calls and audience support

327 The production of 'waa' barks $(\beta \pm \mathrm{SE}=2.59 \pm 1.14, z=2.26, P=0.024)$ but not screams $(\beta \pm \mathrm{SE}=14.57 \pm 1194.19, z=0.01, P=0.990)$ was dependent on whether or not the victim had received support from the audience. Support was usually given by adult or late-adolescent males, who provided $78 \%$ of the recorded instances of support for the victim. When one or more individuals in the third-party audience supported the victim by directing mild or severe aggression at the aggressor, the victim was more likely to produce 'waa' barks. Typically, in cases in which bystanders intervened in the interaction, the victim screamed in response to the original aggressive act until the bystander started to direct aggression at the aggressor, then the victim tended to stop screaming and start 'waa' barking.

\section{$<\mathrm{H} 1>$ Discussion}

Wild chimpanzees that have become victims of physical aggression can utter two basic call types, screams and 'waa' barks. While all utterances are initiated by screams, some of them also contain 'waa' barks after the screams. In our study, we were able to show that the two calls are directed at two different audiences and so serve different social functions.

In particular, the production of screams was influenced by the composition of the third-party audience, indicating that these calls were, at least in part, directed at bystanders. The fact that the number of males but not females was a good predictor of screams might be explained by the fact that males are physically more powerful than females and our results, in line with previous studies (e.g. Slocombe \& Zuberbühler, 2007), indicate that males are more likely than females to provide support for victims. Victims also tended to scream when a higher 
rather than lower ranking male than the aggressor was in close proximity. In this respect, our study is in line with work showing that victims of aggression change the acoustic structure of their screams to exaggerate the level of aggression received if high-ranking individuals are in close proximity (Slocombe \& Zuberbühler, 2007), suggesting that one function of these calls is to solicit help. We did not find evidence that screams are more likely to be given in the presence of affiliated individuals, but this may have been due to fact that friendship patterns between males were unstable during the study period.

In contrast to screams, 'waa' bark production was not dependent on audience composition, and these calls were given after rather than before receiving support from bystanders. Moreover, 'waa' barking victims were visually oriented towards aggressors and likely to retaliate, suggesting that these calls do not function to recruit support from bystanders but to repel the attacker .The ultimate function of 'waa' barking, therefore, may be to discourage the attacker from future aggression. This hypothesis, however, requires further testing, ideally with postconflict data collected over longer timescales. Nevertheless, our interpretation is consistent with the results of a recent experimental study showing that individuals avoid barks of former aggressors' associates (Wittig et al., 2014), suggesting that these calls are aversive to listeners and function to repel them, probably because they reflect an aggressive attitude of the producer.

Concerning the screams, our results suggest that apart from alerting the audience, these calls signal the victim's submission and indicate that he is unlikely to retaliate, which might discourage the aggressor from continuing the assault (e.g. Rowell, 1962). Indeed, vocal sequences consisting of screams only tended to be produced more often when the rank distance between the opponents was large. Both screams and 'waa' barks, therefore, are good 
predictors of the signaller's subsequent behaviour (e.g. Smith, 1977), which may influence the outcome of an aggressive interaction by signalling submission or readiness to retaliate, and by increasing the probability of obtaining support from bystanders.

Our results also suggest that calls are used to manage aggressor-victim relationships following aggression. In particular, 'waa' barks, but not screams, seem to inhibit the occurrence of reconciliation, probably because the aggressor is less likely to approach the victim and reconcile after the victim has 'waa' barked or because victims are unlikely to behave affiliatively towards their aggressors after producing these calls. Owing to the small number of instances of reconciliation following victims' 'waa' barking recorded in this study, we were unable to test between these two hypotheses. None the less, our study suggests that agonistic calls in chimpanzees play an important role in managing relationships between aggressors and victims, including the occurrence of reconciliation, an important element in the sociality of primates and other animals (Aureli \& de Waal, 2000). In baboons, it has been shown that affiliative grunts facilitate reconciliation (Wittig, Crockford, Wikberg, Seyfarth, \& Cheney, 2007), and to our knowledge this is the first study showing that agonistic calls can also influence the likelihood of reconciliation, albeit in the opposite way.

Our study is also relevant for an ongoing debate in the animal communication literature, instigated by Owren and Rendall $(1997 ; 2001)$. Here, the main argument has been that animal vocalizations can have direct physiological effects on recipients, a plausible proposal for both 'waa' barks and screams. Both call types consist of loud and acoustically chaotic sounds which may have direct dissuasive effects on an aggressor (see also Gouzoules \& Gouzoules, 2000). At the same time, however, our results also suggest that screams are primarily directed at third-party audience members, which is inconsistent with an acoustic repellent function. More generally, it has been proposed that the acoustic features of a call are shaped by natural selection in a way that makes the call effective in fulfilling its function (Morton, 1977; Owren 

birds, high-frequency, tonal sounds are often signals of submission, while low-frequency, noisy calls are more likely to be produced by hostile individuals (Hauser, 1993; Morton, 1977; Ordóñez-Gómez et al., 2015). Our results are consistent with this interpretation. In particular, 'waa' barks are lower pitched than screams and victim retaliation was associated with 'waa' barking but not screaming. However, 'waa' barks are given not only during aggressive interactions but also in other dangerous contexts, for example, when encountering bush pigs or other dangerous animals (P. Fedurek, personal observation), probably to repel them. Interestingly, chimpanzees exposed to python models directed their 'waa' barks at preferred social partners that were ignorant about the snake (Schel et al., 2013), as if to drive them away from the danger. Evidence from a range of contexts, therefore, indicates that 'waa' barks function to repel others and, on a proximate level, seem to reflect an individual's confidence and willingness to behave aggressively. Importantly, barking may have a similar function in other species, such as domestic dogs, Canis familiaris (Lord, Feinstein, \& Coppinger, 2009; Yin \& McCowan, 2004), Arctic foxes, Alopex lagopus (Frommolt,

412 Goltsman, \& Macdonald, 2003), roe deer, Capreolus capreolus (Reby, Cargnelutti, \& Hewison, 1999) and sea lions, Zalophus californianus (Schusterman \& Dawson, 1968).

In conclusion, our results show that victim screams and 'waa' barks, although often produced during the same agonistic events and as part of the same vocal sequence, are directed at different types of audiences and fulfil different social functions. 'Waa' barks are signals directed at the aggressor and indicate the probability of retaliation. Screams, on the other hand, are calls primarily directed at the third-party audience to attract support. The use of these two types of calls aids the victim to manage aggressive interactions by signalling either submission or the probability of retaliation, as well as by influencing the probability of reconciliation or receiving support from bystanders. We conclude that agonistic calls play an 
422 important role in mediating agonistic interactions and aggressor-victim relationships

423 following aggression, and that the graded system of chimpanzee vocal production is capable

424 of generating complex signals with multiple functions.

\section{Acknowledgments}

426 We thank the management and staff of the Budongo Conservation Field Station for their support and assistance. We also thank the Uganda Wildlife Authority and the Uganda National Council for Science and Technology for permission to conduct the study. We are grateful to Anne Schel for blind coding the recorded calls and Christof Neumann for his help with Elo-rating calculations. P.F. was funded by Swiss National Science Foundation and European Research Council project grants to K.Z. (PRILANG 283871).

Supplementary Material

Supplementary material associated with this article can be found in the online version at doi.

References

Altmann, J. (1974). Observational study of behaviour: sampling methods. Behaviour, 49(34), 227-267. doi: 10.1163/156853974x00534

Arnold, K., \& Whiten, A. (2001). Post-conflict behaviour of wild chimpanzees (Pan troglodytes schweinfurthii) in the Budongo Forest, Uganda. Behaviour, 138(5), 649690. doi: doi:10.1163/156853901316924520

Aureli, F., Cords, M., \& van Schaik, C. P. (2002). Conflict resolution following aggression in gregarious animals: a predictive framework. Animal Behaviour, 64(3), 325-343. doi: 
Aureli, F., \& de Waal, F. (2000). Natural conflict resolution. Berkeley, CA.: California University Press.

Bernstein, I. S., \& Ehardt, C. L. (1985). Agonistic aiding: kinship, rank, age, and sex influences. American Journal of Primatology, 8(1), 37-52. doi: 10.1002/ajp.1350080105

Bygott, J. D. (1979). Agonistic behaviour, dominance, and social structure in wild chimpanzees of the Gombe National Park. In D. A. Hamburg \& E. R. McCown (Eds.), The great apes (pp. 405-428). Menlo Park, CA: Benjamin/Cummings.

Cairns, S. J., \& Schwager, S. J. (1987). A comparison of association indexes. Animal Behaviour, 35, 1454-1469. doi: 10.1016/s0003-3472(87)80018-0

Cheney, D. (1977). The acquisition of rank and the development of reciprocal alliances among free-ranging immature baboons. Behavioral Ecology and Sociobiology, 2(3), 303-318. doi: 10.1007/BF00299742

Clark AP, Wrangham RW (1994) Chimpanzee arrival pant-hoot- Do they signify food or status? International Journal of Primatology 15:185-205

Crockford, C., \& Boesch, C. (2003). Context-specific calls in wild chimpanzees, Pan troglodytes verus: analysis of barks. Animal Behaviour, 66, 115-125. doi: 10.1006/anbe.2003.2166

de Waal, F., \& Yoshihara, D. (1983). Reconciliation and redirected affection in rhesus monkeys. Behaviour, 85(3), 224-241. doi: doi:10.1163/156853983X00237

Elo, A. E. (1978). The rating of chess players, past and present. New York, NY: Arco.

Fedurek, P., Machanda, Z., Schel, A. M., \& Slocombe, K. E. (2013). Pant hoot chorusing and social bonds in male chimpanzees. Animal Behaviour, 86, 189-196.

Fedurek, P., \& Slocombe, K. E. (2013). The social function of food-associated calls in male chimpanzees. American Journal of Primatology, 75, 726-739. 
Frommolt, K., H., Goltsman, M., E., \& Macdonald, D., W. (2003). Barking foxes, Alopex lagopus: field experiments in individual recognition in a territorial mammal. Animal Behaviour, 65(3), 509-518.

Gilby, I. C., \& Wrangham, R. W. (2008). Association patterns among wild chimpanzees (Pan troglodytes schweinfurthii) reflect sex differences in cooperation. Behavioral Ecology and Sociobiology, 62(11), 1831-1842. doi: 10.1007/s00265-008-0612-6

Goodall, J. (1986). The chimpanzees of Gombe: patterns of behavior. Cambridge, MA: Harvard University Press.

Gouzoules, H., \& Gouzoules, S. (1990). Matrilineal signatures in the recruitment screams of pigtail macaques, Macaca nemestrina. Behaviour, 115(3), 327-347. doi: doi:10.1163/156853990X00635

Gouzoules, H., \& Gouzoules, S. (2000). Agonistic screams differ among four species of macaques: the significance of motivation-structural rules. Animal Behaviour, 59(3), $501-512$

Gouzoules, H., Gouzoules, S., \& Marler, P. (1984). Rhesus monkey (Macaca mulatta) screams: Representational signalling in the recruitment of agonistic aid. Animal Behaviour, 32, 182-193.

Hauser, M. (1993). The evolution of nonhuman primate vocalizations: effects of phylogeny, body weight, and social context. American Naturalist, 142, 528-542.

Hogstedt, G. (1983). Adaptation unto death: function of fear screams. American Naturalist, $121,562-570$.

Kojima, S., Izumi, A., \& Ceugniet, M. (2003). Identification of vocalizers by pant hoots, pant grunts and screams in a chimpanzee. Primates, 44(3), 225-230. doi: 10.1007/s10329002-0014-8 
Lord, K., Feinstein, M., \& Coppinger, R. (2009). Barking and mobbing. Behavioural Processes, 81(3), 358-368.

Marler, P. (1976). Social organization, communication and graded signals: the chimpanzee and the gorilla. In P. P. Bateson \& R. A. Hinde (Eds.), Growing points in ethology (pp. 239-277). Cambridge, U.K.: Cambridge University Press.

Marler, P., \& Tenaza, R. R. (1977). Signalling behavior of apes with special reference to vocalisation. In T. A. Sebeok (Ed.), How animals communicate (pp. 965-1033). Bloomington, IN: Indiana University Press.

McFarland, R., \& Majolo, B. (2013). The importance of considering the behavioral form of reconciliation in studies of conflict resolution. International Journal of Primatology, 34(1), 15-29. doi: 10.1007/s10764-012-9643-y

Mitani, J. C., \& Gros-Louis, J. (1998). Chorusing and call convergence in chimpanzees: Tests of three hypotheses. Behaviour, 135, 1041-1064.

Morton, E. S. (1977). On the occurrence and significance of motivation-structural rules in some bird and mammal sounds. American Naturalist, 111, 855-869.

Muller, M. N., \& Wrangham, R. W. (2004). Dominance, aggression and testosterone in wild chimpanzees: a test of the ' challenge hypothesis '. Animal Behaviour, 67, 113-123. doi: 10.1016/j.anbehav.2003.03.013

Neumann, C., Duboscq, J., Dubuc, C., Ginting, A., Irwan, A. M., Agil, M., . . . Engelhardt, A. (2011). Assessing dominance hierarchies: validation and advantages of progressive evaluation with Elo-rating. Animal Behaviour, 82(4), 911-921.

Newton-Fisher, N. E. (1999). Association by male chimpanzees: a social tactic? Behaviour, 136(6), 705-730. doi: doi:10.1163/156853999501531 
Newton-Fisher, N. E. (2006). Female coalitions against male aggression in wild chimpanzees of the Budongo Forest. International Journal of Primatology, 27(6), 1589-1599. doi: $10.1007 / \mathrm{s} 10764-006-9087-3$

Nieburg, H. L. (1970). Agonistics—rituals of conflict. The Annals of the American Academy of Political and Social Science, 391(1), 56-73. doi: 10.1177/000271627039100106

Ordóñez-Gómez, J. D., Dunn, J. C., Arroyo-Rodríguez, V., Méndez-Cárdenas, M. G., Márquez-Arias, A., \& Santillán-Doherty, A. M. (2015). Role of emitter and severity of aggression influence the agonistic vocalizations of Geoffroy’s spider monkeys (Ateles geoffroyi). International Journal of Primatology, 36(2), 429-440. doi: $10.1007 / \mathrm{s} 10764-015-9833-5$

Owren, M. J., \& Rendall, D. (1997). An affect-conditioning model of nonhuman primate vocal signaling. In D. H. Owings, M. D. Beecher, \& N. S. Thompson (Eds.), Perspectives in Ethology: Vol. 12. Communication (pp. 299-346). New York, NY: Plenum Press.

Owren, M. J., \& Rendall, D. (2001). Sound on the rebound: bringing form and function back to the forefront in understanding nonhuman primate vocal signaling. Evolutionary Anthropology: Issues, News, and Reviews, 10(2), 58-71. doi: 10.1002/evan.1014

Reby, D., Cargnelutti, B., \& Hewison, A. J. M. (1999). Contexts and possible functions of barking in roe deer. Animal Behaviour, 57(5), 1121-1128.

Reynolds, R. (2005). The chimpanzees of Budongo Forest: ecology, behaviour and conservation. Oxford, U.K.: Oxford University Press.

Rohwer, S., Fretwell, S. D., \& Tuckfield, R. C. (1976). Distress screams as a measure of kinship in birds. American Naturalist, 96, 418-430.

Rowell, T. E. (1962). Agonistic noises of the rhesus monkey (Macaca mulatta). Symposia of the Zoological Society of London, 8, 91-96. 
Schel, A. M., Townsend, S. W., Machanda, Z., Zuberbühler, K., \& Slocombe, K. E. (2013). Chimpanzee alarm call production meets key criteria for intentionality. PLoS One, 8(10), e76674. doi: 10.1371/journal.pone.0076674

Schusterman, R. J., \& Dawson, R. G. (1968). Barking, dominance, and territoriality in male sea lions. Science, 160(3826), 434-436. doi: 10.1126/science.160.3826.434

Slocombe, K. E., Kaller, T., Call, J., \& Zuberbühler, K. (2010). Chimpanzees extract social information from agonistic screams. PLoS One, 5(7), e11473. doi: 10.1371/journal.pone.0011473

Slocombe, K. E., Townsend, S. W., \& Zuberbühler, K. (2009). Wild chimpanzees (Pan troglodytes schweinfurthii) distinguish between different scream types: evidence from a playback study. Animal Cognition, 12(3), 441-449. doi: 10.1007/s10071-008-0204-x

Slocombe, K. E., \& Zuberbühler, K. (2005). Agonistic screams in wild chimpanzees (Pan troglodytes schweinfurthii) vary as a function of social role. Journal of Comparative Psychology, 119(1), 67-77. doi: 10.1037/0735-7036.119.1.67

Slocombe, K. E., \& Zuberbühler, K. (2007). Chimpanzees modify recruitment screams as a function of audience composition. Proceedings of the National Academy of Sciences of the United States of America, 104(43), 17228-17233.

Smith, W. J. (1977). The behavior of communicating: An ethological approach. Cambridge, MA: Harvard University Press.

Waller, B. M., Warmelink, L., Liebal, K., Micheletta, J., \& Slocombe, K. E. (2013). Pseudoreplication: a widespread problem in primate communication research. Animal Behaviour, 86(2), 483-488.

Wiley, R. H., \& Richards, D. G. (1978). Physical constraints on acoustic communication in the atmosphere: implications for the evolution of animal vocalizations. Behavioral Ecology and Sociobiology, 3(1), 69-94. doi: 10.1007/bf00300047 
Wittig, R. M., Crockford, C., Langergraber, K. E., \& Zuberbühler, K. (2014). Triadic social interactions operate across time: a field experiment with wild chimpanzees. Proceedings of the Royal Society B: Biological Sciences, 281(1779). doi: 10.1098/rspb.2013.3155

Wittig, R. M., Crockford, C., Wikberg, E., Seyfarth, R. M., \& Cheney, D. L. (2007). Kinmediated reconciliation substitutes for direct reconciliation in female baboons. Proceedings of the Royal Society B: Biological Sciences, 274(1613), 1109-1115. doi: 10.1098/rspb.2006.0203

Yin, S., \& McCowan, B. (2004). Barking in domestic dogs: context specificity and individual identification. Animal Behaviour, 68(2), 343-355.

Zahavi, A. (1979). Why shouting. American Naturalist, 113(1), 155-156.

Appendix

Male preferred social partners

PSPs were established on the basis of three different dyadic association measures. The first measure, simple ratio index (SRI), reflects the total proportion of scans in which both individuals were together in the same party (Cairns \& Schwager, 1987), or

$$
\mathrm{SRI}_{\mathrm{AB}}=\frac{\mathrm{P}_{\mathrm{AB}}}{\mathrm{P}_{\mathrm{A}}+\mathrm{P}_{\mathrm{B}}-\mathrm{P}_{\mathrm{AB}}}
$$

where $P_{\mathrm{AB}}=$ the number of parties containing both $\mathrm{A}$ and $\mathrm{B}, P_{\mathrm{A}}=$ the number of parties containing A, $P_{\mathrm{B}}=$ the number of parties containing $\mathrm{B}$. 
589

590

591

592

593

594

595

596

597

598

599

600

601

602

603

604

605

606

607

608

609

610

611

The second dyadic association measure is the '5 $\mathrm{m}$ association index' (5M) (Gilby \&

Wrangham, 2008) which measures the frequency with which a dyad was observed within $5 \mathrm{~m}$ of one another, given that one of the individuals was present in the party and another one was a focal animal:

$$
5 M_{A B}=\frac{A_{f}\left(B_{5}\right)+B_{f}\left(A_{5}\right)}{A_{f}\left(B_{p}\right)+B_{f}\left(A_{p}\right)}
$$

where $A_{f}\left(B_{5}\right)=$ the number of instances in which $A$ was the focal animal and $B$ was within 5 $\mathrm{m}$ of $\mathrm{A}, \mathrm{B}_{\mathrm{f}}\left(\mathrm{A}_{5}\right)=$ the number of instances in which $\mathrm{B}$ was the focal animal and $\mathrm{A}$ was within 5 $\mathrm{m}, \mathrm{A}_{\mathrm{f}}\left(\mathrm{B}_{\mathrm{p}}\right)=$ the number of instances $\mathrm{A}$ was the focal animal and $\mathrm{B}$ was in the same party, $\mathrm{B}_{\mathrm{f}}\left(\mathrm{A}_{\mathrm{p}}\right)=\mathrm{B}$ was the focal animal and $\mathrm{A}$ was in the same party.

The third employed dyadic association measure is the 'nearest-neighbour association index' (NN) (Gilby \& Wrangham, 2008), which reflects the frequency with which two individuals were observed as nearest neighbours, provided that one was the focal animal and the other was within $5 \mathrm{~m}$, or

$$
\mathrm{NN}_{\mathrm{AB}}=\frac{\mathrm{A}_{\mathrm{f}}\left(\mathrm{B}_{\mathrm{nn}}\right)+\mathrm{B}_{\mathrm{f}}\left(\mathrm{A}_{\mathrm{nn}}\right)}{\mathrm{A}_{\mathrm{f}}\left(\mathrm{B}_{5}\right)+\mathrm{B}_{\mathrm{f}}\left(\mathrm{A}_{5}\right)}
$$

where $A_{f}\left(B_{n n}\right)=$ the number of instances $A$ was the focal animal and $B$ was the nearest neighbour and $B_{f}\left(A_{n n}\right)=$ the number of instances $B$ was the focal animal and $A$ was the nearest neighbour.

For a given index (SRI, 5M and $\mathrm{NN}$ ) individuals $\mathrm{A}$ and $\mathrm{B}$ were classified as 'mutual associates' if the value was one-third of a standard deviation larger than the averages of both A and B. We classified a dyad as mutual preferred social partners (mutual PSP) if they were mutual associates for at least two of the three different indexes (Gilby \& Wrangham, 2008). 
612 Since association dynamics in chimpanzees change on a temporal basis (e.g. Fedurek et al., 613 2013), we conducted association calculations for four separate periods with durations

614 between 4 and 5 months: between June and October 2013, February and May 2014, June and 615 September 2014 and January and April 2015. Using this procedure we identified eight for the 616 first (mean $\pm \mathrm{SD}=1.15 \pm 0.8$ /focal individual, range $0-3$ ), seven for the second (mean $\pm \mathrm{SD}=1.08 \pm 0.76 /$ focal individual, range $0-2$ ), seven for the third (mean $\pm \mathrm{SD}=1.08 \pm 1.32 /$ focal individual, range $0-3$ ) and six (mean $\pm \mathrm{SD}=0.92 \pm 0.95 /$ focal individual, range $0-3$ ) mutual PSP dyads for the fourth period of the study. The remaining dyads were classified as neutral social partners (non-PSPs).

Dominance status

Dominance status was established only for adult and late-adolescent males, using the Elorating procedure. This method is based on a sequence in which interactions between individuals occur rather than on an interaction matrix (Neumann et al., 2011). At the onset of the process each individual is given the same rating of a value 1000. After each agonistic or submissive interaction the score is updated with the winner of the interaction gaining whereas the loser loses points (Neumann et al., 2011). The number of points gained or lost by two interacting individuals is dependent on the expected outcome which in turn depends on previous interactions between these two individuals (Elo, 1978). In our study the scores were based on interactions such as pant grunts (i.e. vocalizations given by males to other males that outrank them) combined with the outcomes of dyadic win-lose agonistic interactions (i.e. physical attack, chase, charge, displacements, etc.; Goodall, 1986; Bygott, 1989; Muller \& Wrangham, 2004) recorded during the study period. Since dominance relationships between male chimpanzees change on a temporal basis (Gilby \& Wrangham, 2008), we calculated 
637 June 2013 and September 2014 and June 2013 and April 2015. The Elo-rating scores were 638 then converted into rank orders for each male (from 1 to 14, with 1 representing the highest 639 ranking male). The Elo-rating method has several advantages over more traditional methods 640 such as sensitivity to short-term demography changes, effectiveness in tracking hierarchy 641 dynamics on short-term scales and more effective evaluation of relative hierarchy position 642 between individuals with undecided interactions (Neumann et al., 2011). We believe that this 643 method was especially effective in establishing dominance positions of the Sonso males, 644 since the hierarchy was unstable throughout the study period with no clear alpha male after 645 one of the males had lost his alpha status prior to the study period. Elo-rating scores were 646 calculated using R v.3.1.1 (The R Foundation for Statistical Computing, Vienna, Austria, 647 http://www.r-project.org). 
650 Figure Legends

651 Figure 1. An example time-frequency spectrogram of an utterance consisting of $(a, b)$ two

652 screams followed by (c, d) two waa barks given by an adult male.

653 Figure 2. The relationship between the mean number of males present in close proximity to

654 the victim and whether or not the victim produced screams (GLMM; $* P \leq 0.05$; random

655 effects: aggressor ID and victim ID; error bars represent 1 SD).

656 Figure 3. The relationship between 'waa' bark production and retaliation (GLMM; * $P \leq 0.05$;

657 random effects: aggressor ID and victim ID).

658 Figure 4. The relationship between 'waa' bark production and reconciliation (GLMM;

$659 * * P \leq 0.01$; random effects: aggressor ID and victim ID).

660

661

662 
Table 1. Summary of the data set examined

\begin{tabular}{|c|c|c|c|c|}
\hline Age-sex category of aggressor-victim & $\begin{array}{l}\text { Aggression } \\
\text { bouts }\end{array}$ & $\begin{array}{l}\text { Bouts of severe } \\
\text { aggression }\end{array}$ & $\begin{array}{l}\text { Bouts with } \\
\text { screams }\end{array}$ & $\begin{array}{l}\text { Bouts } \\
\text { 'waa' }\end{array}$ \\
\hline Adult male-adult male & 85 & 22 & 68 & 26 \\
\hline Adult male - late-adolescent male & 33 & 5 & 33 & 7 \\
\hline Adult male-adult female & 61 & 18 & 58 & 14 \\
\hline Adult male - late-adolescent female & 5 & 3 & 5 & 0 \\
\hline $\begin{array}{l}\text { Adult male-early adolescent male or } \\
\text { female }\end{array}$ & 6 & 0 & 5 & 1 \\
\hline Late adolescent male-adult male & 8 & 2 & 5 & 3 \\
\hline $\begin{array}{l}\text { Late adolescent male - late-adolescent } \\
\text { male }\end{array}$ & 4 & 2 & 2 & 1 \\
\hline Late adolescent male-adult female & 16 & 4 & 14 & 4 \\
\hline $\begin{array}{l}\text { Late adolescent male - late-adolescent } \\
\text { female }\end{array}$ & 1 & 0 & 1 & 0 \\
\hline $\begin{array}{l}\text { Late adolescent male-early adolescent } \\
\text { male or female }\end{array}$ & 1 & 0 & 1 & 0 \\
\hline Adult female-adult male & 2 & 2 & 2 & 0 \\
\hline Adult female-adult female & 1 & 0 & 1 & 0 \\
\hline Total & 223 & 58 & 195 & 56 \\
\hline
\end{tabular}

664

665 The table gives details of the number of aggression bouts and the number of bouts that

666 included severe aggression, screams or 'waa' barks produced by the victim, retaliation by the

667 victim or reconciliation between the interacting animals that we recorded for each of the age-

668 sex categories of the aggressor and victim. 
670 Table 2. The relationship between the production of screams and various types of audience

671 (independent variables)

672

673

Independent variables

Coefficient SE

$95 \%$ confidence interval

$\begin{array}{lllllll}\text { Number of males within } 15 \mathrm{~m} & 0.58 & 0.26 & 2.25 & 0.024 & 0.07 & 1.08\end{array}$

Number of females within 15

0.18

0.37

0.49

0.622

$-0.55$

0.91

$\mathrm{m}$

Total number of males in party

$-0.08$

0.13

$-0.61$

0.542

$-0.33$

0.17

Total number of females in

0.16

0.17

0.93

0.354

$-0.18$

0.50

party

674

675 GLMM; Dependent variable: scream (0/1); Random effects: aggressor ID and victim ID.

676 
677 Table 3. The relationship between 'waa' barking and various types of audience (independent

678 variables)

$\begin{array}{llllll}\text { Independent variables } & \text { Coefficient } & \mathrm{SE} & z & P & 95 \% \text { confidence interval }\end{array}$

\begin{tabular}{lllllll}
\hline Number of males within $15 \mathrm{~m}$ & 0.09 & 0.10 & 0.85 & 0.393 & -0.11 & 0.29 \\
Number of females within 15 & -0.15 & 0.15 & -1.01 & 0.310 & -0.43 & 0.14 \\
$\mathrm{~m}$ & & & & & & \\
Total number of males in party & -0.01 & 0.08 & -0.09 & 0.929 & -0.17 & 0.15 \\
& & & & & & \\
Total number of females in & 0.11 & 0.10 & 1.10 & 0.271 & -0.08 &
\end{tabular}

679

680 GLMM; Dependent variable: 'waa' bark (0/1); Random effects: aggressor ID and victim ID.

681

682 